AIAA-2004-1528

\title{
A NOVEL ACTUATED COMPOSITE TAPE-SPRING FOR DEPLOYABLE STRUCTURES
}

\author{
Thomas W. Murphey* \\ Sergio Pellegrino ${ }^{\dagger}$ \\ University of Cambridge, Cambridge, CB2 1PZ, UK
}

\begin{abstract}
$\underline{\text { Abstract }}$
This paper presents a novel composite tape-spring that is suitable for use as a structural element of deployable space structures. The tape-spring uses a combination of material orthotropy and prestress to achieve the unique property known as neutral stability. A consequence of this property is that the tape-springs can be partially rolled and they neither want to unroll or rollup. Previously investigated composite tape-springs always proceed towards their manufactured geometry once the rolling process has been started. This paper documents a procedure to fabricate neutrally stable tape-springs. An analysis methodology is presented that predicts neutral stability in appropriately prestressed laminates. The analysis predictions are shown to agree with observations. Finally, the ability of neutrally stable tape-springs to undergo large elastic deformations with diminishingly small forces is exploited in the development of actuated tape-springs. Analytical and experimental results are presented.
\end{abstract}

\section{Introduction}

This paper presents a novel composite tape-spring that is suitable for use as a structural element of deployable space structures. The tape-spring has the unique property that it is neutrally stable; as shown in the sequence of photographs in Figure 1, the tapespring is static in a continuum of positions without external forces to hold it. This class of structure is referred to here as a Neutrally Elastic Mechanism (NEM) because its behavior is functionally equivalent to typical sliding contact joint mechanisms. A consequence of this neutral stability is that diminishingly small forces are required to roll or unroll the tape-spring. The tape-spring can therefore be

\footnotetext{
*Engineer, Dynacs Military \& Defense, Inc., Member AIAA, Thomas . murphey@Kirtland .af.mil

${ }^{\dagger}$ Professor of Structural Engineering, University of

Cambridge, Associate Fellow AIAA,

pellegrino@eng.cam.ac.uk

Copyright (C) 2004 by Thomas W. Murphey. Published by the American Institute of Aeronautics, Inc. with permission.
}

actuated (controlled rolling and unrolling) with relatively small and low force actuators bonded to the tape-spring surface. Two actuation options are investigated here: NiTi shape memory allow and PVDF piezoelectric film.

Such actuated NEM tape-springs offer four prominent features for deployable space structures. First, they allow controlled deployment. Similarly, they allow controlled retraction. Third, their neutral stability allows for zero stiffness isolators and actuators. Finally, they allow mass efficient (with respect to stiffness and strength) structures in the fully deployed configuration. Several planar deployable structures that have potential to benefit from NEM tape-springs have recently been investigated; Lockheed Martin is developing a rolled solar array ${ }^{1}$, and JPL has investigate various structures with rolled tubes. These structures could be constructed from actuated NEM tape-spring frames for a lightweight, controlled deployment system. The tape-springs could also be used in controlled deployment trusses. A third application is vibration isolators. The neutral stability of NEM tape-springs enables an actuator with mechanical continuity (no sliding parts) and theoretically zero stiffness. A final application is flex harnessing. Elastic high conductivity beryllium-copper wires could be configured in a NEM tape-spring such that there is low parasitic deployment torque, regardless of temperature.

This paper presents a description of NEM tapespring construction, an analytical model of the tapesprings based on classical laminate theory, and finally, experimental results from efforts to actuate NEM tapesprings with NiTi shape memory alloy and PVDF piezoelectric film bonded to the tape-spring surface.

NEM tape-springs achieve their unique properties through a material pre-stress, similar to how metal tapesprings have been made to exhibit interesting properties with engineered pre-stresses. ${ }^{2}$ As will be shown, the orthotropic materials under investigation here allow for stable deformations not possible with isotropic materials. The current tape-springs are in marked contrast to the bi-stable composite tape-springs that have been of recent interest. ${ }^{3-5}$ Such tape-springs are 
not neutrally stable; when partially rolled, they tend toward their stress-free manufactured configuration (typically unrolled) with great force and release of strain energy. Neutrally stable tape-springs are neither stress-free in the fully rolled or fully unrolled configurations. They are engineered so that both the rolled and unrolled configurations store equal amounts of strain energy.

\section{Neutrally Stable Tape-Spring Construction}

A fiber reinforce plastic (FRP) composite neutrally stable tape-spring can be constructed from a cross-ply laminate where each lamina individually has a curvature pre-stress, as shown in Figure 2. The two curved lamina, with perpendicular curvature axes and opposing curvature senses, are bonded together in a flat configuration. The resulting tape-spring in stable when fully rolled or completely unrolled and it is neutrally in partially rolled (transition) configurations. Neutrally stable tape-springs are not limited to this construction method (extensional pre-stress and more complicated lamina stacking sequences can also be used to). A cross-ply laminate is employed here for its simplicity.

Through several iterations, a laboratory scale fabrication procedure was developed that yields short lengths of neutrally stable tape-springs. This procedure can be divided into three sub-procedures: curved lamina fabrication, coiled lamina fabrication and lamina bonding. For this research, a $198 \mathrm{~g} / \mathrm{m}^{2}$ Hexcel T300 graphite fiber and epoxy resin system prepreg was used. The Hexcel published mechanical properties of this lamina are listed in Table 1.

Table 1: Hexcel Fibredux 914 TS(6K)-5-34\% (unidirectional T300 graphite fiber/epoxy prepreg with $34 \%$ resin content by weight) mechanical properties.

\begin{tabular}{|c|c|c|}
\hline Description & Symbol & Value \\
\hline Cured Thickness & $t$ & $0.13 \mathrm{~mm}$ \\
\hline Fiber Direction Modulus & $E_{1}$ & $125.0 \mathrm{GPa}$ \\
\hline Transverse Modulus & $E_{2}$ & $7.5 \mathrm{GPa}$ \\
\hline Shear Modulus & $G$ & $6.3 \mathrm{GPa}$ \\
\hline Poisson's Ratio & $\nu$ & 0.33 \\
\hline
\end{tabular}

\section{Curved Lamina Fabrication}

A $3.2 \mathrm{~cm}$ diameter mandrel is mold release treated. One lamina is placed directly on the mandrel with fibers in the circumferential direction. A $0.02 \mathrm{~mm}$ thick phosphorus-bronze shim stock caul plate is mold release treated and taped in place directly over the fibers. The prepared part is vacuum bagged, autoclave cured and removed from the mandrel. A finished transversely curved lamina is shown in Figure 3 with mandrel and caul plate.
Bagging plies that provide paths for air and resin to bleed off are typically included in more traditional composites fabrication techniques. These plies better consolidate a laminate (reduced voids) and provide more uniform fiber distribution. These plies are not used here because they also introduce surface and thickness non-uniformities. Curing directly on the mandrel and caul plate ensures the most constant thickness and blemish-free part.

\section{Coiled Lamina Fabrication}

Fabrication of long axially curved laminas is problematic because the fibers must be coiled continuously over itself as tape is wrapped on spool. A long and narrow (width equal to desired tape-spring width) mold release treated caul plate is taped perpendicularly to the mandrel. The prepreg (fibers in the long direction) material is placed on the caul plate and under tension, the caul plate and fibers are wrapped around the mandrel (Figure 4). The caul plate is taped in place when wrapping is finished. Heat shrink tape is then wrapped around the part to provide curing consolidation pressure. The part is oven cured and removed from the mandrel.

It is important that vacuum bagging and autoclave pressure are not used in this step. All attempts by the authors at doing so resulted in crimped laminas as shown in Figure 5. It appears that spaces between wraps in the coils are initially present or develop during the curing process. The extreme pressures of an autoclave cure seek the smallest volume part, which is created when one ridge bulges out from the mandrel. In contrast, heat shrink tape provides a uniform pressure that maintains the desired circular shape even if gaps are present in the spiral.

\section{Lamina Bonding}

In this step, the laminas are bonded together in a flat configuration. Both the curved and coiled laminas are taped to flat caul plates as shown in Figure 6. The exposed surfaces are sanded with fine sand paper and cleaned with acetone. A light coating of epoxy (same as used in prepreg) is applied to both surfaces. The surfaces are mated, vacuum bagged and cured.

There are two options for assembling the curved and coiled laminas. The laminas can be bonded such that they open away from each other (Figure 2) or they can be bonded to such that they open towards each other. Once bonded, both configurations appear identical. However, no methods we found to successfully bond the laminas as shown in Figure 2. In this configuration, delamination cracks easily propagate since the two laminas are curved such that they want to separate from each other. The results presented in this paper are for tape-springs bonded with facing openings. In this configuration cracks do not propagate since the laminas are pushed against each other. 
Several neutrally stable tape-springs have been fabricated using the above difficult and tedious procedure. It is envisioned that the process could be automated using continuous cure processes similar to the pultrusion industry.

\section{Pre-Stressed Tape-Spring Analysis}

An extension of classical laminate theory $\left(\mathrm{CLT}^{8}\right)$ is now presented for the stability analysis of tape-springs. The method assumes the tape-springs are constructed from plates that are:

i) initially flat,

ii) large or thin enough that edge effects are negligible,

iii) uniformly pre-stressed.

Studies have shown that such laminates primarily deform into flat or cylindrical configurations. ${ }^{2-3,6-7}$ Any other configuration (such as a non-zero guasian curvature surface) requires significant stretching strain to accommodate the bending. As a result, cylindrical surfaces store less strain energy and are the preferred configurations.

A linear analysis of such laminates using CLT is adequate in that it inherently accommodates large cylindrical deformations. However, CLT does not directly reveal their equilibrium configurations and stability. The method developed here accomplishes this by assuming the deformations are cylindrical and calculating the stored strain energy for a range of enforced deformations. Applying the principal of minimum potential energy, a search is then made for the enforced cylindrical deformations that are strain energy minimums and hence, the equilibrium configurations. The minima are analyzed for their stability characteristic.

Lamina Pre-stresses. Lamina prestresses are specified as strains with respect to the specific reference configuration of a flat plate with only moment edge loads (surface or normal edge loads are not permitted). The strains in this configuration are assumed to be linear through the thickness of a lamina, but are allowed to be discontinuous across the laminate as shown in Figure 7. Two vectors per lamina are required to specify the strain state. The vectors of mid-plane strains, $\quad \varepsilon_{k}^{o, i}=\left[\varepsilon_{1}^{o, i}, \varepsilon_{2}^{o, i}, \gamma_{12}^{o, i}\right]_{k}^{\mathrm{T}}$, and the vectors of curvatures, $\quad \boldsymbol{\kappa}_{k}^{i}=\left[\kappa_{1}^{i}, \kappa_{2}^{i}, \kappa_{12}^{i}\right]_{k}^{\mathrm{T}}$, in lamina principal material coordinates are employed here (superscript $i$ indicates an initial pre-strain and a subscript $k$ refers to lamina $k$ ). The pre-strain state must satisfy the following integral to ensure normal edge loads are zero,

$$
\int_{z_{n}}^{z_{0}} \bar{\sigma} d z=0
$$

where $\bar{\sigma}$ is the vector of plane stresses in laminate coordinates. In the following section, CLT is reviewed because it will be used to calculate the laminate stored strain energy.

Classical Laminate Theory. Mid-plane strain and curvature vectors transform from lamina principal material coordinates (indicated by subscript 1 and 2 ) to laminate coordinates (indicated by subscript $x$ and y) according to,

$$
\overline{\boldsymbol{\varepsilon}}_{k}^{o, i}=\left[\begin{array}{c}
\varepsilon_{x}^{o, i} \\
\varepsilon_{y}^{o, i} \\
\gamma_{x y}^{o, i}
\end{array}\right]_{k}=\mathbf{T}_{k}\left[\begin{array}{c}
\varepsilon_{1}^{o, i} \\
\varepsilon_{2}^{0, i} \\
\gamma_{12}^{o, i}
\end{array}\right]_{k}
$$

and,

$$
\overline{\boldsymbol{\kappa}}_{k}^{i}=\left[\begin{array}{c}
\boldsymbol{\kappa}_{x}^{i} \\
\boldsymbol{\kappa}_{y}^{i} \\
\boldsymbol{\kappa}_{x y}^{i}
\end{array}\right]_{k}=\mathbf{T}_{k}\left[\begin{array}{c}
\boldsymbol{\kappa}_{1}^{i} \\
\boldsymbol{\kappa}_{2}^{i} \\
\boldsymbol{\kappa}_{12}^{i}
\end{array}\right]_{k}
$$

where,

$$
\mathbf{T}_{k}=\left[\begin{array}{ccc}
\cos ^{2} \theta_{k} & \sin ^{2} \theta_{k} & -\cos \theta_{k} \sin \theta_{k} \\
\sin ^{2} \theta_{k} & \cos ^{2} \theta_{k} & \cos \theta_{k} \sin \theta_{k} \\
2 \cos \theta_{k} \sin \theta_{k} & -2 \cos \theta_{k} \sin \theta_{k} & \cos ^{2} \theta_{k}-\sin ^{2} \theta_{k}
\end{array}\right]
$$

An over-bar indicates a vector or matrix is with respect to laminate coordinates. $\theta_{k}$ is the angular orientation of the lamina relative to the laminate, measured positive from the $x$ towards the $y$ axis in a right-handed coordinate system with $z$ pointing down. Engineering shear strain is used in the above equations and is defined as,

$$
\gamma_{x y}=\frac{\partial u}{\partial y}+\frac{\partial v}{\partial x}
$$

and the twist curvature is defined as,

$$
\kappa_{x y}=-2 \frac{\partial^{2} w}{\partial x \partial y}
$$

where $u, v$ and $w$ are the $x, y$ and $z$ displacements, respectively.

Strain is assumed to be related to stress through plane-stress Hooke's law for an orthotropic material,

$$
\boldsymbol{\sigma}=\mathbf{Q} \boldsymbol{\varepsilon}
$$

where,

$$
\boldsymbol{\sigma}=\left[\begin{array}{c}
\sigma_{1} \\
\sigma_{2} \\
\tau_{12}
\end{array}\right], \quad \mathbf{Q}=\left[\begin{array}{ccc}
Q_{11} & Q_{12} & 0 \\
Q_{12} & Q_{22} & 0 \\
0 & 0 & Q_{66}
\end{array}\right] \quad \text { and } \quad \boldsymbol{\varepsilon}=\left[\begin{array}{c}
\varepsilon_{1} \\
\varepsilon_{2} \\
\gamma_{12}
\end{array}\right]
$$

In terms of engineering material constants, the reduced stiffness terms $Q_{i j}$ are given by,

$$
\begin{gathered}
Q_{11}=\frac{E_{1}}{1-v_{12} v_{21}}, Q_{12}=\frac{v_{12} E_{2}}{1-v_{12} v_{21}}, \\
Q_{22}=\frac{E_{2}}{1-v_{12} v_{21}} \text { and } Q_{66}=G_{12}
\end{gathered}
$$

Through the reciprocity relation 


$$
v_{21}=\frac{E_{2}}{E_{1}} v_{12}
$$

Stress transforms to laminate coordinates according to,

$$
\begin{aligned}
\overline{\boldsymbol{\sigma}}_{k} & =\left[\begin{array}{c}
\sigma_{x} \\
\sigma_{y} \\
\tau_{x y}
\end{array}\right]_{k} \\
& =\left[\begin{array}{ccc}
\cos ^{2} \theta_{k} & \sin ^{2} \theta_{k} & -2 \cos \theta_{k} \sin \theta_{k} \\
\sin ^{2} \theta_{k} & \cos ^{2} \theta_{k} & 2 \cos \theta_{k} \sin \theta_{k} \\
\cos \theta_{k} \sin \theta_{k} & -\cos \theta_{k} \sin \theta_{k} & \cos ^{2} \theta_{k}-\sin ^{2} \theta_{k}
\end{array}\right]\left[\begin{array}{l}
\sigma_{1} \\
\sigma_{2} \\
\tau_{12}
\end{array}\right]_{k}
\end{aligned}
$$

The $\mathbf{A}$ and $\mathbf{B}$ stiffness matrices of CLT and are given by

$$
\begin{aligned}
& \mathbf{A}=\sum_{k=1}^{n} \overline{\mathbf{Q}}_{k}\left(z_{k}-z_{k-1}\right) \\
& \mathbf{B}=\frac{1}{2} \sum_{k=1}^{n} \overline{\mathbf{Q}}_{k}\left(z_{k}^{2}-z_{k-1}^{2}\right)
\end{aligned}
$$

$\overline{\mathbf{Q}}_{k}$ are the lamina reduced stiffness matrices in laminate coordinates and are given by

$$
\overline{\mathbf{Q}}_{k}=\left[\begin{array}{lll}
\bar{Q}_{11} & \bar{Q}_{12} & \bar{Q}_{16} \\
\bar{Q}_{12} & \bar{Q}_{22} & \bar{Q}_{26} \\
\bar{Q}_{16} & \bar{Q}_{26} & \bar{Q}_{66}
\end{array}\right]_{k}
$$

where,

$$
\begin{aligned}
\bar{Q}_{11}= & Q_{11} \cos ^{4} \theta+2\left(Q_{12}+2 Q_{66}\right) \sin ^{2} \theta \cos ^{2} \theta \\
& +Q_{22} \sin ^{4} \theta \\
\bar{Q}_{12} & =\left(Q_{11}+Q_{22}-4 Q_{66}\right) \sin ^{2} \theta \cos ^{2} \theta \\
& +Q_{12}\left(\sin ^{4} \theta+\cos ^{4} \theta\right) \\
\bar{Q}_{22}= & Q_{11} \sin ^{4} \theta+2\left(Q_{12}+2 Q_{66}\right) \sin ^{2} \theta \cos ^{2} \theta+ \\
& Q_{22} \cos ^{4} \theta \\
\bar{Q}_{16}= & \left(Q_{11}-Q_{12}-2 Q_{66}\right) \sin \theta \cos ^{3} \theta \\
& +\left(Q_{12}-Q_{22}+2 Q_{66}\right) \sin { }^{3} \theta \cos \theta \\
\bar{Q}_{26}= & \left(Q_{11}-Q_{12}-2 Q_{66}\right) \sin { }^{3} \theta \cos \theta \\
& +\left(Q_{12}-Q_{22}+2 Q_{66}\right) \sin \theta \cos 3 \\
\bar{Q}_{66}= & \left(Q_{11}+Q_{22}-2 Q_{12}-2 Q_{66}\right) \sin ^{2} \theta \cos ^{2} \theta \\
& +Q_{66}\left(\sin ^{4} \theta+\cos ^{4} \theta\right)
\end{aligned}
$$

Enforced Cylindrical Deformations. As shown in Figure 7, total strains (c) in a prestressed laminate with enforced cylindrical deformations are the sum of prestrains (a) and strains due to the enforced deformation (b). The enforced strains are that due to a cylindrical deformation with arbitrary curvature $\left(\kappa_{e}\right)$ and axis orientation $\left(\theta_{e}\right)$. With respect to the laminate coordinate system these curvatures are,

$$
\overline{\boldsymbol{\kappa}}_{e}=\kappa_{e}\left[\begin{array}{c}
\sin ^{2} \theta_{e} \\
\cos ^{2} \theta_{e} \\
-2 \sin \theta_{e} \cos \theta_{e}
\end{array}\right]
$$

Laminates which are not symmetric or anti-symmetric generally exhibit coupling between bending and stretching so that the enforced curvatures also induce laminate mid-plane strains. The laminate mid-plane strains that result from the enforced deformations are

$$
\overline{\boldsymbol{\varepsilon}}^{o, e}=-\mathbf{A}^{-1} \mathbf{B} \overline{\mathbf{\kappa}}_{e}
$$

In laminate coordinates the lamina enforced mid-plane strains are given by

$$
\bar{\varepsilon}_{k}^{o, e}=\bar{\varepsilon}^{o, e}+\frac{1}{2}\left(z_{k}+z_{k-1}\right) \overline{\mathrm{k}}_{e}
$$

As illustrated in Figure 7 (c), the enforced strains are added to the pre-strains to give the total lamina midplane strains and curvatures

$$
\begin{aligned}
& \overline{\boldsymbol{\varepsilon}}_{k}^{o, T}=\overline{\boldsymbol{\varepsilon}}_{k}^{o, i}+\overline{\boldsymbol{\varepsilon}}_{k}^{o, e} \\
& \overline{\mathbf{\kappa}}_{k}^{T}=\overline{\mathbf{\kappa}}_{k}^{i}+\overline{\mathbf{\kappa}}_{e}
\end{aligned}
$$

The strain energy density (strain energy per area) of a deformed pre-stressed laminate is found by summing the strain energy in each lamina ${ }^{9}$,

$$
u=\frac{1}{2} \sum_{k=1}^{n}\left(\left[\boldsymbol{\varepsilon}_{k}^{o, T}\right]^{\mathrm{T}} \mathbf{A}_{k} \boldsymbol{\varepsilon}_{k}^{o, T}+\left[\overline{\mathbf{\kappa}}_{k}^{T}\right]^{\mathrm{T}} \mathbf{D}_{k} \overline{\mathrm{K}}_{k}^{T}\right)
$$

The lamina stiffness matrices are given by

$$
\begin{aligned}
& \mathbf{A}_{k}=t_{k} \overline{\mathbf{Q}}_{k} \\
& \mathbf{D}_{k}=\frac{t_{k}^{3}}{12} \overline{\mathbf{Q}}_{k} .
\end{aligned}
$$

Equilibrium and Stability Analysis. According to the principle of minimum potential energy, equilibrium configurations exist when the instantaneous rate of change of strain energy density with respect to all deformation modes is zero. The magnitude of the maximum rate of change in strain energy density with respect to any deformation mode is given by the magnitude of the gradient of the strain energy density function $^{9}$,

$$
|\operatorname{grad} u|=\sqrt{\left(\frac{\partial u}{\partial \kappa_{e}}\right)^{2}+\left(\frac{\partial u}{\partial \theta_{e}}\right)^{2}} .
$$

The conditions for this maximum to be zero and, thus, an equilibrium point are

$$
\frac{\partial u}{\partial \kappa_{e}}=0 \text { and } \frac{\partial u}{\partial \theta_{e}}=0 .
$$

Solutions to Equations (22) are the equilibrium points within the currently assumed cylindrical deformation manifold.

The stability characteristics of the equilibrium points are revealed through analysis of the structural stiffness with respect to small deformations from these points. The second directional derivative with respect 
to a deformation mode is the instantaneous stiffness $\left(K_{\psi}\right)$ of the deformation mode

$$
K_{\psi}=D_{\mathbf{b}}\left(D_{\mathbf{b}} u\right)=\mathbf{b} \cdot \operatorname{grad}(\mathbf{b} \cdot \operatorname{grad} u)
$$

where $\mathbf{b}$ is the unit vector in the direction of the derivative. All cylindrical deformation modes can be described by combinations of the two parameters $\kappa_{e}$ and $\theta_{e}$ and can be expressed as

$$
\mathbf{b}=\left[\begin{array}{c}
\cos \psi \\
\sin \psi
\end{array}\right]
$$

where $\psi$ is the angular orientation of $\mathbf{b}$ and ranges $\pm \pi$. The components of $\mathbf{b}$ represent the $\kappa_{e}$ and $\theta_{e}$ directions and have units of 1/length and radians, respectively. Expanding Equation (23) with Equation (24) and writing the result in matrix form gives the stiffness in the $\psi$ direction

$$
K_{\psi}=\mathbf{b}^{\mathrm{T}} \mathbf{K b}
$$

where

$$
\mathbf{K}=\left[\begin{array}{cc}
\frac{\partial^{2} u}{\partial \kappa_{e}^{2}} & \frac{\partial^{2} u}{\partial \kappa_{e} \partial \theta_{e}} \\
\frac{\partial^{2} u}{\partial \kappa_{e} \partial \theta_{e}} & \frac{\partial^{2} u}{\partial \theta_{e}^{2}}
\end{array}\right] .
$$

The minimum stiffness, $K_{\min }$, determines the stability characteristics of an equilibrium point. Three cases can arise:

$$
\begin{aligned}
& K_{\min }>0 \rightarrow \text { stable } \\
& K_{\min }=0 \rightarrow \text { neutrally stable } \\
& K_{\min }<0 \rightarrow \text { unstable }
\end{aligned}
$$

Energy wells correspond to the first case. They are stable and deformation in any direction from that point is resisted. Equilibrium points where the minimum stiffness is zero are neutrally stable. Such points have at least one deformation mode with zero stiffness for infinitesimally small deformations. If neighboring points are also neutrally stable then a neutrally elastic path exists. Energy peaks, ridges or saddles correspond to the case of instability; structures can be quasi-stably balanced at these points, but the slightest deviation and they tend toward a lower energy configuration.

Equation (25) is quadratic and, therefore, two criteria for stability $\left(K_{\min }>0\right)$ are ${ }^{10}$,

$$
\frac{\partial^{2} u}{\partial \kappa_{e}^{2}}>0 \text { and } \frac{\partial^{2} u}{\partial \kappa_{e}^{2}} \frac{\partial^{2} u}{\partial \theta_{e}^{2}}-\left(\frac{\partial^{2} u}{\partial \kappa_{e} \partial \theta_{e}}\right)^{2}>0 .
$$

The criteria for neutral stability $\left(K_{\min }=0\right)$ are

$$
\frac{\partial^{2} u}{\partial \kappa_{e}^{2}} \geq 0 \text { and } \frac{\partial^{2} u}{\partial \kappa_{e}^{2}} \frac{\partial^{2} u}{\partial \theta_{e}^{2}}-\left(\frac{\partial^{2} u}{\partial \kappa_{e} \partial \theta_{e}}\right)^{2}=0 .
$$

The criteria for instability $\left(K_{\min }<0\right)$ are either

$$
\frac{\partial^{2} u}{\partial \kappa_{e}^{2}}<0
$$

or

$$
\frac{\partial^{2} u}{\partial \kappa_{e}^{2}} \frac{\partial^{2} u}{\partial \theta_{e}^{2}}-\left(\frac{\partial^{2} u}{\partial \kappa_{e} \partial \theta_{e}}\right)^{2}<0 .
$$

Cross-ply Laminate Analysis. Consider a prestressed cross-ply laminate constructed as described in the previous section ( $\theta_{1}=0$ and $\theta_{2}=90$ degrees) with pre-stress curvatures in the reference state given by

$$
\boldsymbol{\kappa}_{1}^{i}=\left[\begin{array}{lll}
-\kappa_{i} & 0 & 0
\end{array}\right]^{\mathrm{T}} \quad \text { and } \quad \boldsymbol{\kappa}_{2}^{i}=\left[\begin{array}{lll}
\kappa_{i} & 0 & 0
\end{array}\right]^{\mathrm{T}} .
$$

where $\kappa_{i}=62.5 \mathrm{~m}^{-1}$. The extensional pre-stresses associated with flattening the curved laminas to the flat reference configuration are zero. For the material properties given in Table 1 , the cylindrical deformation strain energy density contour plot is shown in Figure 8. There are four equilibrium points, the properties of which are listed in Table 2. Two of the points are stable and two are unstable (corresponding to a flat laminate). The stable shapes have opposite curvatures, they are aligned with the laminate coordinate axes and they are of larger radius than each lamina was alone.

\section{Table 2: Cross-ply laminate analysis results.}

\begin{tabular}{|c|c|c|c|c|c|}
$\begin{array}{c}\kappa_{e} \\
\left(\mathrm{~m}^{-1}\right)\end{array}$ & $\begin{array}{c}\theta_{e} \\
(\mathrm{rad})\end{array}$ & $\begin{array}{c}u \\
\left(\mathrm{~J} / \mathrm{m}^{2}\right)\end{array}$ & $\begin{array}{c}\text { Criteria 1 } \\
(\mathrm{J})\end{array}$ & $\begin{array}{c}\text { Criteria 2 } \\
(\mathrm{J} / \mathrm{m} / \mathrm{rad})\end{array}$ & Stability \\
\hline 35.3 & 0 & 60.5 & $0.0372>0$ & $4.58>0$ & stable \\
35.3 & $\frac{\pi}{2}$ & 60.5 & $0.0372>0$ & $4.58>0$ & stable \\
0 & $\frac{\pi}{4}$ & 83.8 & $0.0246>0$ & $-6.93<0$ & unstable \\
0 & $-\frac{\pi}{4}$ & 83.8 & $0.0246>0$ & $-6.93<0$ & unstable
\end{tabular}

The analysis predicts the laminate to have stable equilibrium curvatures of $35.3 \mathrm{~m}^{-1}$ and the manufactured laminate curvatures were measured to be $28.1 \mathrm{~m}^{-1}$, a $26 \%$ difference.

\section{NEM Tape-Spring Actuation}

A strain energy analysis characterizes the behavior of partially rolled tape-spring actuators. Consider the three tape-spring sections: rolled, transition and unrolled. With motion of the transition section, one end of the tape-spring lengthens while the other equally shortens. The transition section remains of constant length and therefore, the strain energy in the transition section does not change. The change in strain energy in 
the end sections is only a function of the strain energy densities in the unrolled $\left(u_{u}\right)$ and rolled $\left(u_{r}\right)$ configurations and the distance the transition section is translated ( $d$, positive as the length of the rolled section increases). The change in strain energy with actuation is,

$$
\Delta_{U}=d w\left(u_{r}-u_{u}\right)
$$

where $w$ is the flattened width of the tape-spring. For positive $\Delta_{U}$, energy must be added to roll the tapespring; unabated, the tape-spring will unroll. For negative $\Delta_{U}$, the stored strain energy decreases with rolling; unabated, the tape-spring will roll up.

The force (applied without friction at any point on the rolled tape-spring and parallel to the straight section) required to stop motion is that which does just enough work to compensate for the change in stored strain energy,

$$
F=w\left(u_{r}-u_{u}\right)
$$

The special neutrally stable case exists when there is no change in strain energy between configurations $\left(u_{r}-u_{u}=0\right)$. In such cases, no force is required to stop motion and, conversely, the smallest of forces will induce motion. Such NEM tape-springs have greater potential for ease of actuation and efficiency as this configuration requires the smallest amount of energy to be generated by the actuation material and motion is not resisted by the stiffness of the actuator material.

Equation (34) gives the blocking force, a parameter often used to characterize the performance of an actuator. A second often used parameter to characterize actuators is stress-free length change. In transition actuation, the length change is limited by the length of the tape-spring and, in theory, could be as long as desired.

The rolling nature of transition actuation severely limits the space available to place actuator materials because materials of significant thickness will increase the rolled tube diameter. A laminate type actuator (with thickness comparable to the tape-spring) is need, such that it may be laminated to either or both sides of the tape-spring. A thin film type actuator would minimally add to the rolled tape-spring diameter. Two classes of actuator materials are considered here: piezoelectric polymers and nickel-titanium shape memory alloy. Both actuators have two important deformation mechanisms. First, they have an actuation mechanism that changes the stress-free dimensions of the material. Second, they are essentially elastic materials that obey isotropic or orthotropic elasticity models. Consideration of both deformation mechanisms is required to model these actuator materials.

Regardless of the specific actuator material employed, it is proposed that the deformation mechanics can be sufficiently modeled as initial prestresses analogous to a material coefficient of thermal expansion and associated temperature change $(T)$,

$$
\boldsymbol{\varepsilon}_{a}=T \boldsymbol{\alpha}
$$

where (engineering shear strain is used),

$$
\boldsymbol{\alpha}=\left[\begin{array}{c}
\alpha_{1} \\
\alpha_{2} \\
0
\end{array}\right]
$$

The method presented in the previous section is now used calculate the strain energy densities in rolled and unrolled actuated tape-springs and their ensuing blocking forces. The actuated tape-spring is assumed to be constructed from the cross-ply laminate described herein. It is then laminated on both sides with an actuator material of thickness $t_{a}$. The tradition of specifying the strains in the reference configuration of a flat plate with no edge loads is continued here. As such, the specified strain state (including actuation strains) must satisfy Equation (1). It is assumed the actuator material is bonded to a flat tape-spring (the reference configuration) un-actuated and stress free. Ensuing actuation of the actuator materials then changes this strain state in such a way that Equation (1) remains satisfied. Thus, the un-actuated reference strain state is given by Equation (32) where all extensional strains are zero and the actuator curvature strains are also zero. For simplicity, it is here assumed that the actuation laminas are actuated in equal and opposite directions such that in the reference configuration they induce no extensional strains and Equation (1) is inherently satisfied. With these assumptions and assuming the actuation strains are isotropic, the top and bottom actuation layer initial strains are given by,

$$
\bar{\varepsilon}_{\text {top }}^{o, i}=\left[\begin{array}{c}
\varepsilon_{a} \\
\varepsilon_{a} \\
0
\end{array}\right] \text { and } \bar{\varepsilon}_{\text {bot }}^{o, i}=\left[\begin{array}{c}
-\varepsilon_{a} \\
-\varepsilon_{a} \\
0
\end{array}\right]
$$

The described strain energy density analysis was carried out numerically for both PVDF and NiTi shape memory alloy. The predicted blocking forces are a linear function of the actuation strain. This allows definition of a blocking force coefficient as,

$$
C_{\text {block }}=\frac{F}{\varepsilon_{a}}
$$

The blocking force coefficient is a strong function of the actuator layer thickness. If the layer is too thin, there is not enough material to have any effect. If the layer is to thick, its bending stiffness inhibits actuation.

Analysis results are shown in Figure 9 for PVDF ( $E=3.0 \mathrm{GPa}$ ). An optimal actuator layer thickness exists and corresponds to $0.243 \mathrm{~mm}$. This thickness gives a maximum blocking force coefficient of $533 \mathrm{~N} / \varepsilon$. PVDF has a maximum actuation strain of 
approximately 0.0006, indicating the maximum blocking force obtainable is $0.32 \mathrm{~N}$. At this optimal thickness and maximum actuation strain, the strain energy densities are $87.0 \mathrm{~J} / \mathrm{m}^{2}$ in the rolled configuration and $81.1 \mathrm{~J} / \mathrm{m}^{2}$ in the unrolled configuration. This is only a $7 \%$ difference and indicates that the tape-springs will be sensitive to manufacturing imperfections.

Analysis results are shown in Figure 10 for NiTi shape memory alloy ( $E=83.0 \mathrm{GPa}$ in the hot state). An optimal actuator layer thickness exists and corresponds to $0.060 \mathrm{~mm}$, much thinner than for PVDF. This thickness gives a maximum actuation coefficient of $1035 \mathrm{~N} / \varepsilon$, twice that of PVDF. NiTi has a maximum repeatable actuation strain of approximately 0.04 , indicating the maximum blocking force obtainable is 41.4 N. However, inspection of the equilibrium points indicates that at a very small strain of 0.00059 , the laminate loses its bi-stable characteristics (the analysis predicts the rolled state to actually be flat). At this strain, the maximum blocking force is $0.61 \mathrm{~N}$ and the strain energy densities are $95.2 \mathrm{~J} / \mathrm{m}^{2}$ in the rolled configuration and $83.9 \mathrm{~J} / \mathrm{m}^{2}$ in the unrolled configuration. This is a $12 \%$ difference and indicates that the NiTi actuated tape-springs will be slightly less sensitive to manufacturing imperfections than the PVDF actuated tape-springs.

\section{Experimental Results}

Experiments were hindered by the difficulty in fabricating high quality neutrally stable tape-springs. Laminate bonding proved sensitive to unknown factors and a reliable bonding procedure was not developed. Small quantities of NEM tape-springs were produced, however, they exhibit noticeable imbalances such that they tend to either roll or unroll. Local variations in the lamina pre-stress curvatures also cause bumps in the otherwise smooth rolling of the tapes. These bumps caused the tape-springs to have a somewhat jerky behavior as the tape-spring snaps between bumps.

$110 \mu \mathrm{m}$ thick PVDF film (Measurement Specialties, Product \#3-1004346-0), silver ink coated on both sides, was laminated to both sides of the described NEM tape-springs. Attempts at actuating the tape-spring with an applied voltage of $300 \mathrm{~V}$ were unsuccessful. The curvature of the tape-spring changed by approximately $4 \%$ during the experiment, however, the desire transition actuation motion was not observed.

Trained NiTi shape memory alloy ribbons were laminated to the convex side of a NEM tape-spring. The ribbons strips were $0.11 \mathrm{~mm}$ thick and $7 \mathrm{~mm}$ wide (for a smeared out thickness of $0.029 \mathrm{~mm}$ ). The NiTi strips were successful in transition actuating the tapesprings. However, the motion was not strong and was jerky due to non-uniformities in the underlying tapespring.

\section{Conclusions}

A new concept for neutrally stable tape-spring has been presented. An analysis methodology predicting and characterizing this neutral stability was developed. Tape-springs were fabricated and shown to exhibit the desired neutral stability. The analysis methodology was use to predict the behavior of tape-springs with PVDF and NiTi shape memory alloy actuator materials laminated to them. The analysis predicted maximum actuation forces on the order of $0.5 \mathrm{~N}$. Actuated neutrally stable tape-springs show potential for use in low force, high displacement actuators. Analytical predictions indicate they can generate small, though significant forces and travel great distances. Unfortunately, NEM tape-springs are inherently difficult to manufacture and sensitive to process controls. Successful use of NEM tape-springs will require future research into more controlled and ideally automated fabrication procedures.

\section{References}

1 Winter, J. et al., “A Proposed Large Deployable Space Structures Experiment for High Power, Large Aperture Missions in MEO,” IEEE 2004 Aerospace Conference.

2 Kebadze, E., Guest, S. D. and Pellegrino, S. P., "Bistable Prestressed Shell Structures"

3 Iqbal, K. and Pellegrino, S., "Bi-stable composite shells,” In Proc. 41st

AIAA/ASME/ASCE/AHS/ASC Structures, Structural Dynamics, and Materials Conference and Exhibit, 3-6 April 2000, Atlanta, GA, USA.

4 Galletly, D.A. and Guest, S.D. (2000). "Equilibrium and Stability Analysis of Composite Slit Tubes." In Proc. IASS-IACM 2000 Fourth International Colloquium on Computation of Shell and Spatial Structures, 5-7 June 2000, Chania, Crete.

5 Guest, S. D., “An inextensional model for bistable slite tubes”.

6 Hyer, M. W., "Calculations of the RoomTemperature Shapes of Unsymmetric Laminates.” Journal of Composite Materials, July 1981, pp. 296310.

7 Dano, M.-L. and Hyer, M.W., “ Thermally-Induced Deformation Behavior of Unsymmetric Laminates," International Journal of Solids and Structures, vol. 35, no. 17, pp. 2101-2120, 1998 .

8 Jones, R. M., Mechanics of Composite Materials, $2^{\text {nd }}$ Ed. Taylor \& Francis, Inc., 1999, Philadelphia, $\mathrm{Pa}$. 
9 Mansfield, E. H., "The Bending and Stretching of Plates, $2^{\text {nd }} E d$. Cambridge University Press, Cambridge, 1989.
10 Strang, G. "Introduction to Applied Methematics," Wellesley-Cambridge Press, 1986.

a)

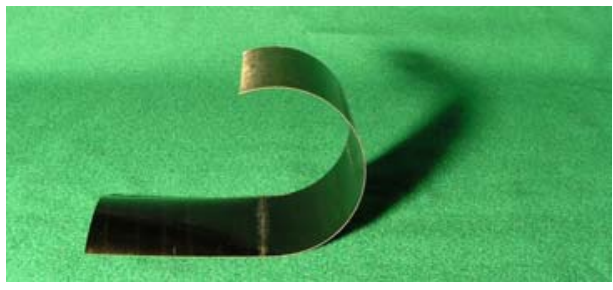

b)

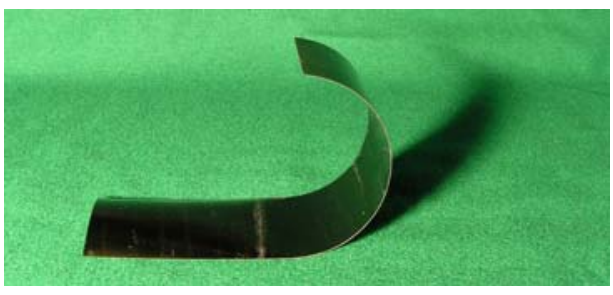

c)

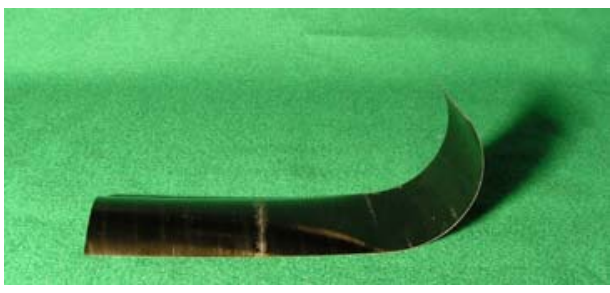

d)

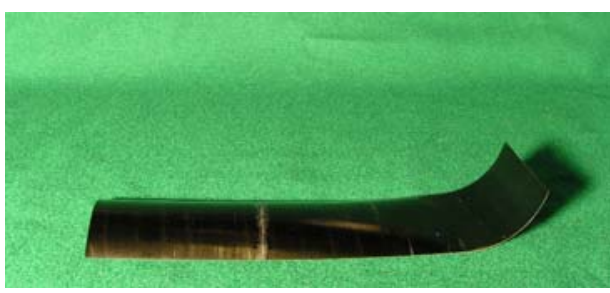

Figure 1: The continuum of neutrally stable configurations in a NEM tape-spring.

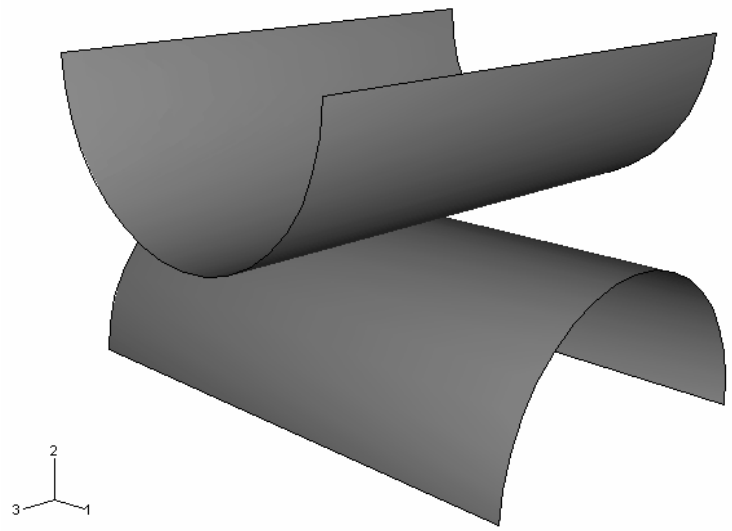

Figure 2: Lamina curvature pre-stresses and orientations prior to bonding flat. 


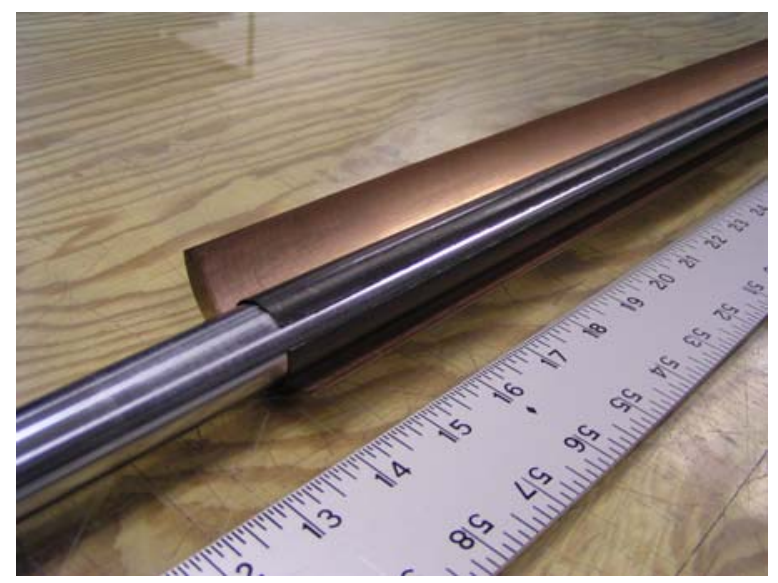

Figure 3: Cured curved lamina mandrel and caul plate.

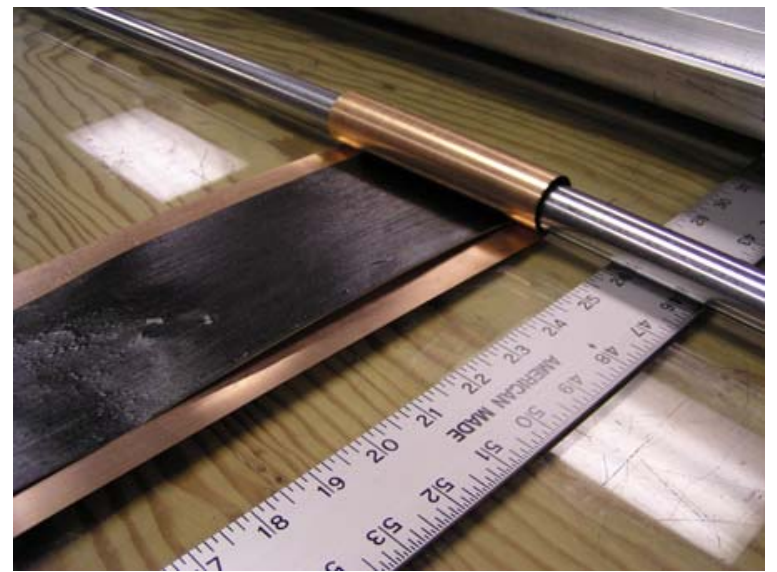

Figure 4: Coiled lamina mandrel and caul plate.

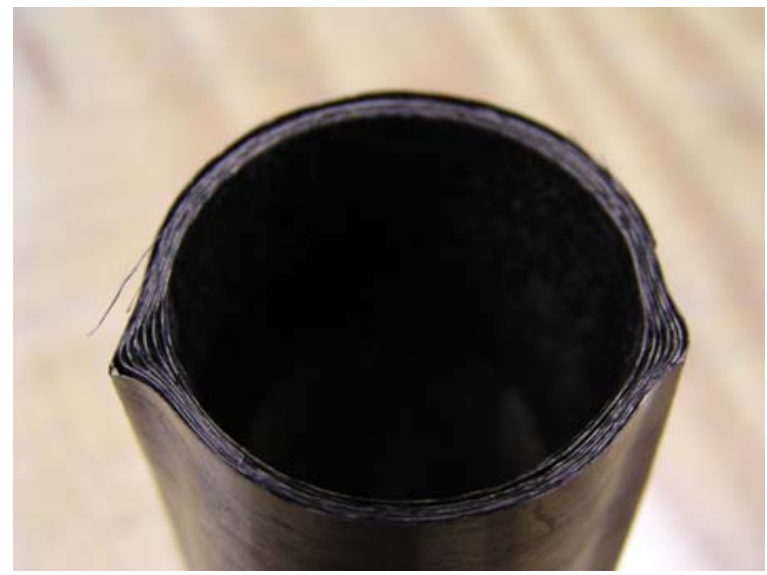

Figure 5: Crimped coiled lamina as a result of autoclave and vacuum bag pressures. 


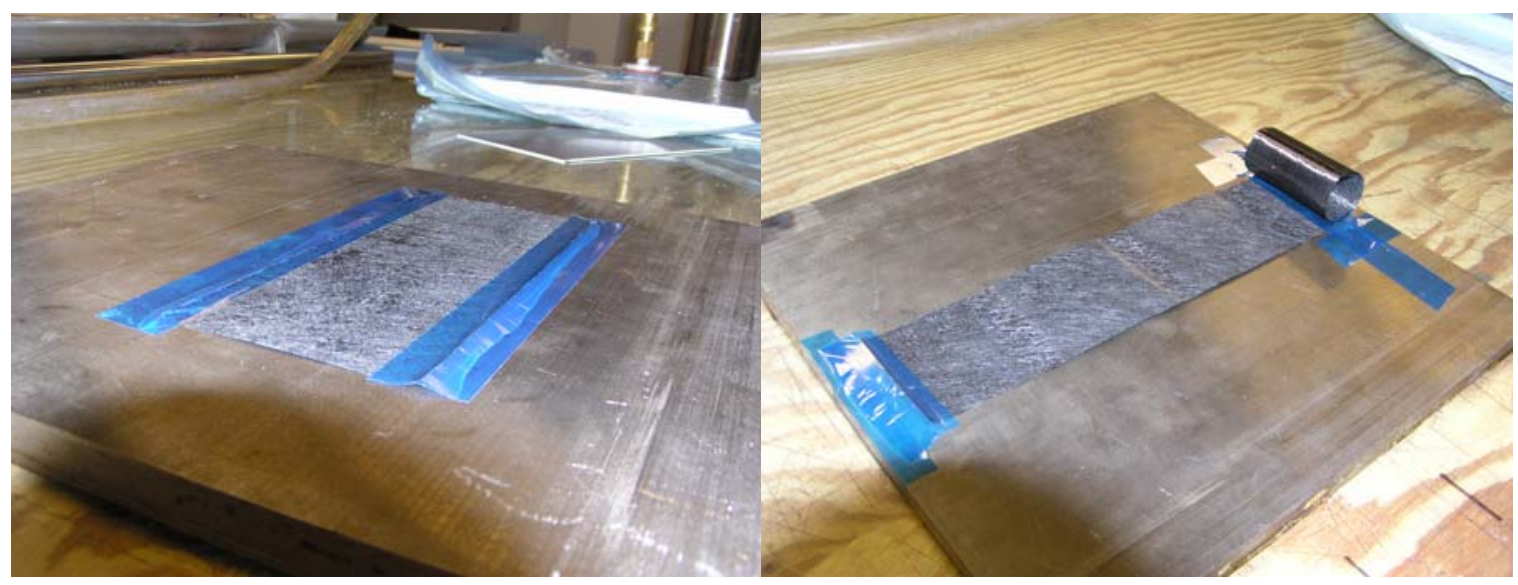

Figure 6: Coiled and curved laminas taped to caul plates in preparation for bonding.

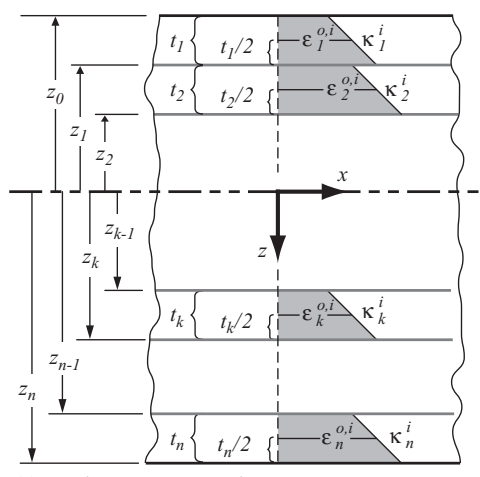

(a) Lamina pre-stress strains

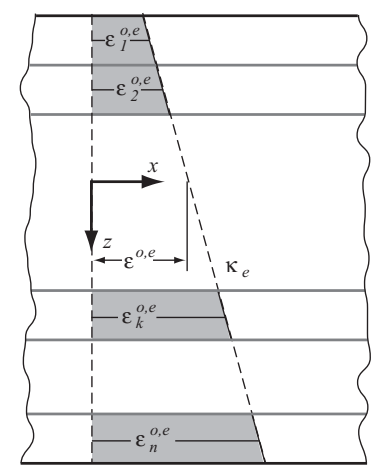

(b) Strains due to cylindrical deformations

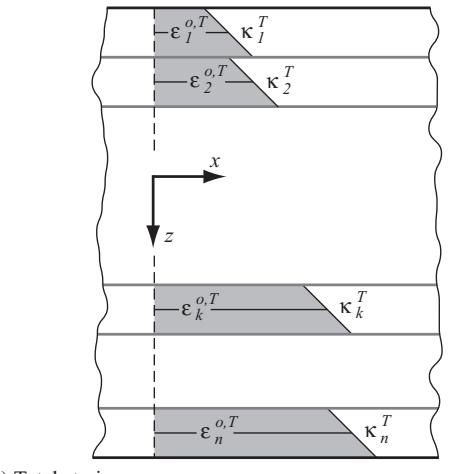

(c) Total strains

Figure 7: Specification of pre-strains in an arbitrary laminate. 


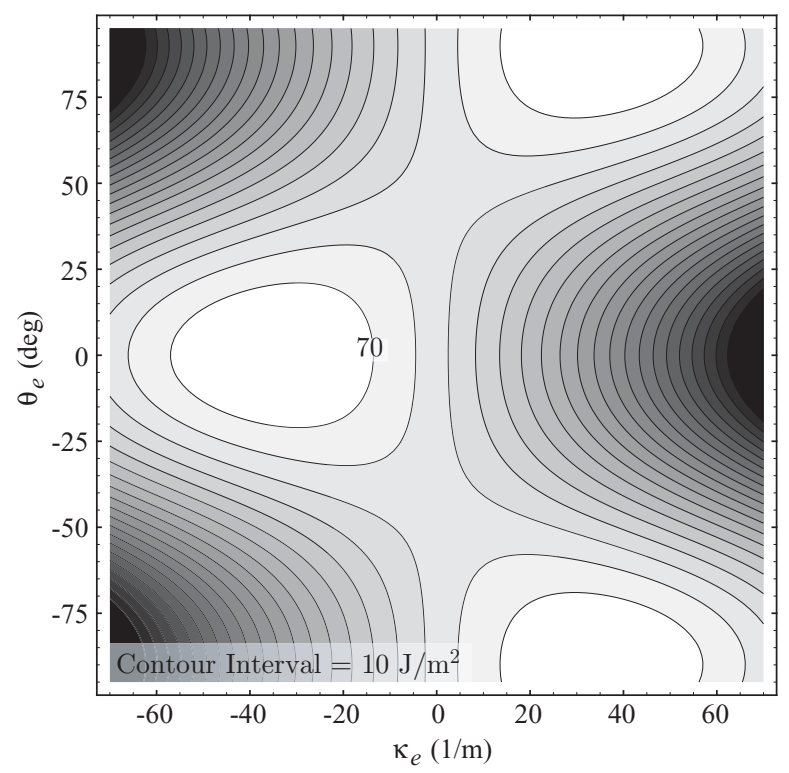

Figure 8: Strain energy density as a function of cylindrical deformations for an opposite sense curvature prestressed cross-ply laminate.

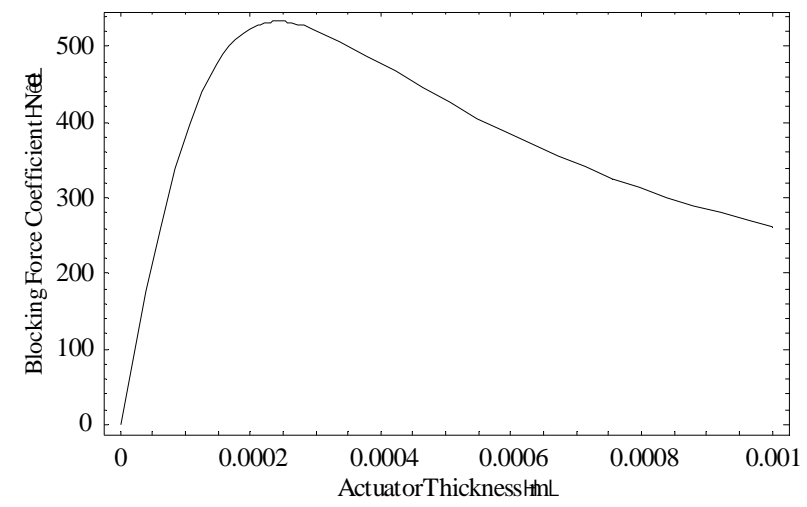

Figure 9: Blocking coefficient as a function of actuator material thickness from bi-axially stretched PVDF.

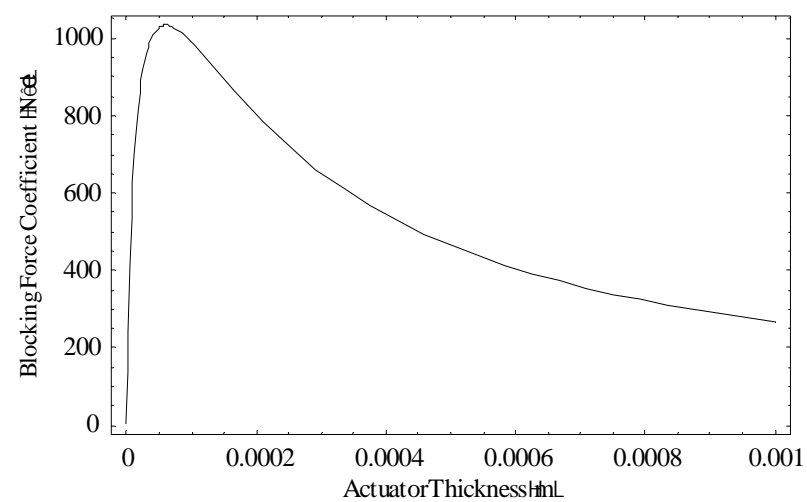

Figure 10: Blocking coefficient as a function of actuator material thickness from NiTi shape memory alloy. 